\section{Image Diagnosis - Interrupted Aortic Arch in a Child With Differential Cyanosis -}

Haisong Bu, MD; Xueyang Gong, MD

A 7-year-old Chinese boy presenting with cyanosis and moderate exercise-induced shortness of breath patent ductus arteriosus (PDA) closure. On physical examination, differential oxygen saturation was noted in the upper and lower extremities of $97 \%$ and $85 \%$, respectively. Blood pressure in the arms was $118 / 90 \mathrm{mmHg}$ and that in the legs was $96 / 68 \mathrm{mmHg}$. A loud P2 was audible at the second left intercostal space. Echocardiogram from the local county hospital showed a large PDA. Computed tomography angiography showed an interrupted aortic arch (IAA; Figure A-D; arrows), aortic discontinuity distal to the left subclavian artery (type A; Figure B-D), and PDA (Figure A,B,E; arrows). Descending aorta angiography confirmed an IAA with non-visualization of 3 branches of the aortic arch and PDA (Figure F; arrow). Right heart catheterization indicated severe fixed pulmonary hyperten- sion with a mean pulmonary artery pressure of $84 \mathrm{mmHg}$ and pulmonary vascular resistance of 8 Woods units. Soon after the patient was then referred to anatomic correction.

This report highlights the importance of increasing the preoperative diagnosis rate in Chinese rural areas. Failure to identify IAA in this report of attempted PDA closure would have led to catastrophic lower-half ischemia.

\section{Acknowledgment}

We thank the patient for participating in this study.

\section{Disclosures}

The authors declare no conflicts of interest.

Received January 9, 2019; accepted January 10, 2019; J-STAGE Advance Publication released online February 19, 2019 Time for primary review: 1 day

Department of Cardiovascular Surgery, Second Xiangya Hospital, Central South University, Changsha, Hunan, China

Mailing address: Xueyang Gong, MD, Department of Cardiovascular Surgery, Second Xiangya Hospital, Central South University, 139 Renmin Central Road, Changsha, Hunan 410011, China. E-mail: gongxueyang@csu.edu.cn

ISSN-1346-9843 All rights are reserved to the Japanese Circulation Society. For permissions, please e-mail: cj@j-circ.or.jp
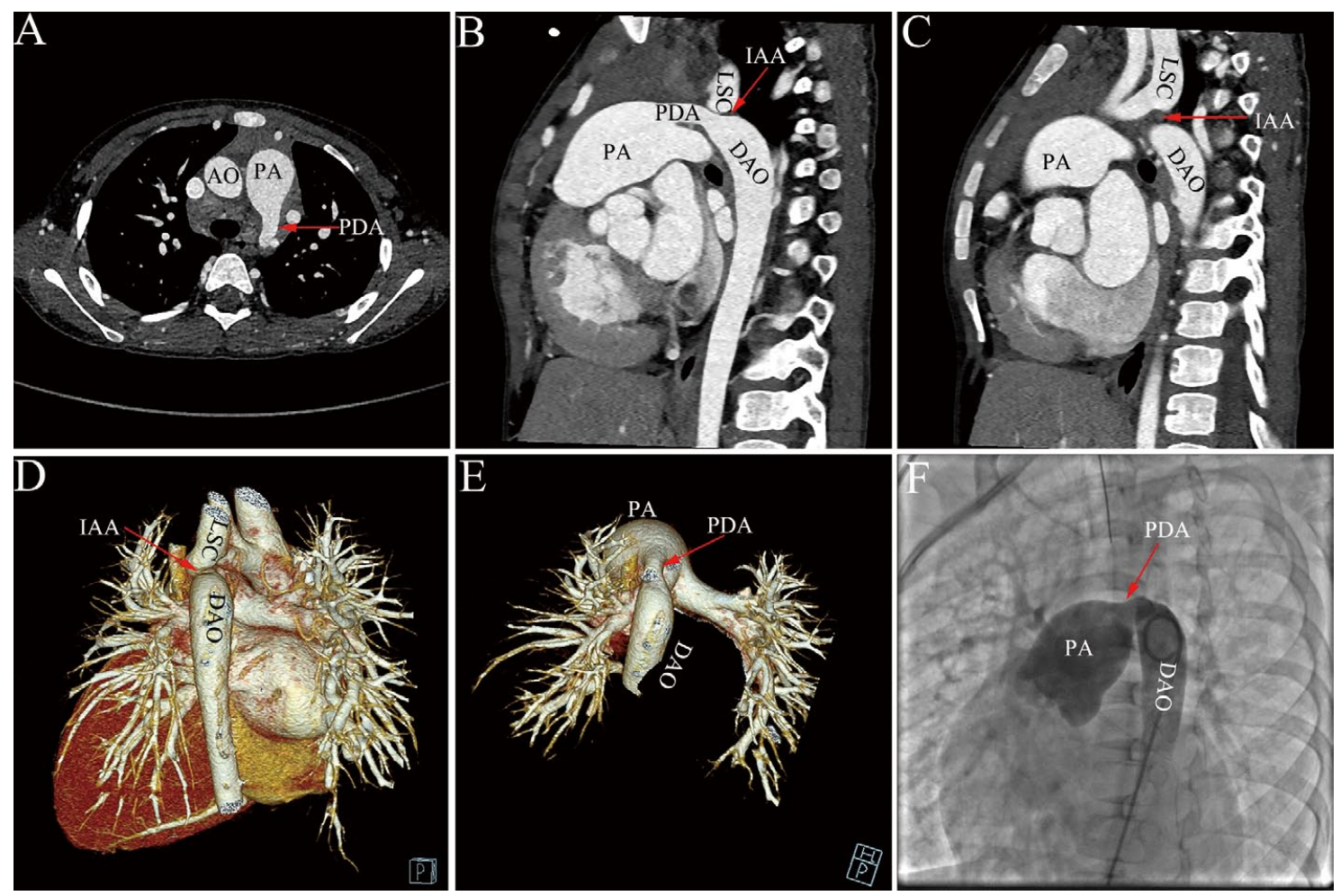

Figure. Imaging of interrupted aortic arch (IAA). (A-C) Computed tomography (CT) showing aortic discontinuity distal to the left subclavian artery and a patent ductus arteriosus (PDA). (D,E) Angio-CT reconstruction of the PDA and IAA. (F) Descending aortic angiography demonstrating an IAA with non-visualization of 3 branches of the aortic arch. AO, aorta; DAO, descending aorta; LSC, left subclavian artery; PA, pulmonary artery. 